\title{
Dramatic localized electromagnetic enhancement in plasmon resonant nanowires
}

\author{
Jörg P. Kottmann ${ }^{\text {a }}$, Olivier J.F. Martin ${ }^{\mathrm{a}, *}$, David R. Smith ${ }^{\mathrm{b}}$, Sheldon Schultz ${ }^{\mathrm{b}}$ \\ a Electromagnetic Fields and Microwave Electronics Laboratory, Swiss Federal Institute of Technology, ETH-Zentrum, \\ 8092 Zurich, Switzerland \\ b Department of Physics, University of California, San Diego, 9500 Gilman Drive, La Jolla, CA 92093-0319, USA
}

Received 6 December 2000; in final form 31 January 2001

\begin{abstract}
We investigate numerically the plasmon resonances of 10-50 nm nanowires with a non-elliptical section. Such wires have a much more complex behavior than elliptical wires and their resonances span a larger frequency range. The field distribution at the surface of these wires exhibits a dramatic enhancement, up to several hundred times the incident field amplitude. These strongly localized fields can provide an important mechanism for surface enhanced Raman scattering (SERS). (C) 2001 Elsevier Science B.V. All rights reserved.
\end{abstract}

\section{Introduction}

The interaction of light with small metal particles has been of great interest, ever since Faraday recognized that gold colloids are responsible for the wide color range of stained glass. Faraday noted 'that a mere variation in the size of its particles gave rise to a variety of colors' [1]. The interest in colloidal metals increased dramatically with the discovery of the phenomenon of surface enhanced Raman scattering (SERS), wherein the Raman signal of molecules ensembles adsorbed on rough metal surfaces can be enhanced by a factor of $10^{7}$ [2-5]. In recent experiments, Raman enhancement up to $10^{12}$ were even reported for single molecules located on so-called 'hot spots' [6-8].

\footnotetext{
${ }^{*}$ Corresponding author. Fax: +41-1-632-1647.

E-mail address: martin@ifh.ee.ethz.ch (O.J.F. Martin).
}

The adsorption of the molecule on the metal can participate in the Raman enhancement $[9,10]$. However, it is believed that the excitation of plasmons in the metallic nanoparticles creates greatly enhanced local electromagnetic fields that contribute the major component of the SERS effect. Motivated by experiments on relatively large systems, recent theoretical efforts have been devoted to the understanding of plasmon resonances in aggregated or periodic metallic systems [11-14].

Recent experiments indicate that SERS can also be observed on individual or small clusters of nanoparticles [6-8]. However, not all similarly sized particles produce equivalent enhancements, and there appears to be a very significant shape or morphology dependence. It is thus essential to further our understanding of the behavior of plasmon resonances for isolated nanoparticles with arbitrary shape. Unfortunately, a complete description of the plasmon resonances of such 
particles is not easy to obtain. Analytic methods are only known for very simple shapes, like spherical or ellipsoidal particles [15], and we must resort to numerical methods for more complex geometries. The resonances of cubic particles, for example, have been investigated in the electrostatic limit by Fuchs [16], while Jensen et al. [17] studied large truncated tetrahedra in the $100 \mathrm{~nm}$ range.

\section{Model}

In this Letter, we investigate metal nanoparticles in the $50 \mathrm{~nm}$ range, as these have been shown both to be individually observable by optical microscopy $[18,19]$ and to individually exhibit the SERS effect $[6,7]$. Here, we concentrate on twodimensional particles (wires) with triangular crosssections, which exhibit strong field enhancements and - in spite of their simple shape - display a wide variety of complex behavior. A more systematic investigation of different particle shapes will be presented in another publication.

Nanowires can be fabricated in a controlled manner in the sub-100 $\mathrm{nm}$ range. Used as substrate, they produce a strong enhancement for SERS [20,21] and fluorescence [22]. Further, metallic nanowires sustaining plasmon resonances have become a very active research field over the last few years, with implications in optics and solid state physics $[18,23,24]$

Throughout the Letter we consider silver wires, illuminated by plane waves with the incident electric field in the plane of the figures (transverse electric polarization). For the permittivity $\varepsilon(\lambda)$ as a function of the wavelength $\lambda$ we use the bulk data of Johnson and Christy [25]. Note that particles down to $10 \mathrm{~nm}$ can well be modeled using bulk permittivity, while smaller particles require a modified value of $\varepsilon(\lambda)$, with - in the case of silver a larger imaginary part than the bulk [26].

Plasmons prove difficult to accurately characterize by numerical methods, because they generally have spatial variations of field and charge density on a scale much smaller than the wavelength of the light which excites them. We address these problems using a newly developed technique based on the integral equation

$$
\mathbf{E}(\mathbf{r})=\mathbf{E}^{0}(\mathbf{r})+\int \mathrm{d} \mathbf{r}^{\prime} \mathbf{G}^{\mathrm{B}}\left(\mathbf{r}, \mathbf{r}^{\prime}\right) k_{0}^{2}\left(\varepsilon\left(\mathbf{r}^{\prime}\right)-\varepsilon^{\mathrm{B}}\right) \mathbf{E}\left(\mathbf{r}^{\prime}\right)
$$

for the electric field $\mathbf{E}(\mathbf{r})$ scattered by a particle, described by $\varepsilon(\mathbf{r})$, illuminated with the incident field $\mathbf{E}^{0}(\mathbf{r})$. The dyadic $\mathbf{G}^{\mathrm{B}}\left(\mathbf{r}, \mathbf{r}^{\prime}\right)$ is the Green's tensor associated with the homogeneous background $\varepsilon^{\mathrm{B}}$. Contrary to the standard Green's tensor technique or the coupled dipole approximation $[14,17]$, we use finite elements to solve Eq. (1); i.e., we develop the unknown field $\mathbf{E}(\mathbf{r})$ on a series of linear basis functions defined on triangular elements and apply a Galerkin scheme to obtain a system of algebraic equations from Eq. (1). We refer the reader to [27], where this technique is described in detail.

To assess the accuracy of this approach, in Fig. 1 we compare the scattering cross-section for an elliptical wire with that obtained using Mie theory [15]. The agreement between the two is excellent: both the position and the amplitude of the resonances are accurately reproduced with our technique. Such an ellipse has two different resonances that can be excited independently for the two illumination directions considered here. For incidence in a direction off either of the principal axes, both resonances are excited.

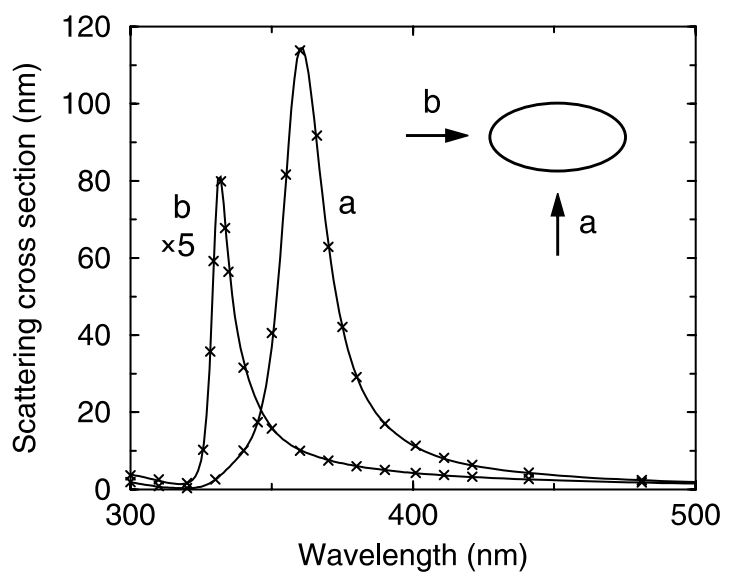

Fig. 1. Accuracy of the method: scattering cross-section for an elliptical particle (overall size $40 \mathrm{~nm} \times 20 \mathrm{~nm}$ ) computed with finite elements (solid line) and with Mie theory (cross). Two orthogonal propagation directions for the incident field, corresponding to two different resonances, are considered. 


\section{Results}

In Fig. 2 we present the scattering cross-section for a triangular particle. Dealing with sharp corners introduces additional numerical difficulties since the field becomes singular at short distances from an infinitely sharp, perfectly conducting corner [28]. However, the sharpness of a real particle is limited by surface and boundary energies; therefore, we have rounded off each corner by 0.25 $\mathrm{nm}$, providing a more realistic model and removing the numerical instabilities. The results shown in Fig. 2 were obtained with 3000 triangular elements. We verified that both 2000 and 4000 discretization elements produced the same numerical results (within better than $1 \%$ ).

The behavior observed in Fig. 2, with up to four different resonances, depending on the illumination direction, is much more complex than that in Fig. 1. The triangle resonances now cover a larger wavelength range, from 330 to $412 \mathrm{~nm}$. (For the ellipse the two resonances are at 331 and $358 \mathrm{~nm}$, respectively.)

For larger particles, the scattering cross-section increases and the different resonances are redshifted (Fig. 3). This shift is actually larger for triangular particles compared to elliptical particles: the main resonance in Fig. 3 shifts from 401 to $427 \mathrm{~nm}$ when the particle size increases from 10

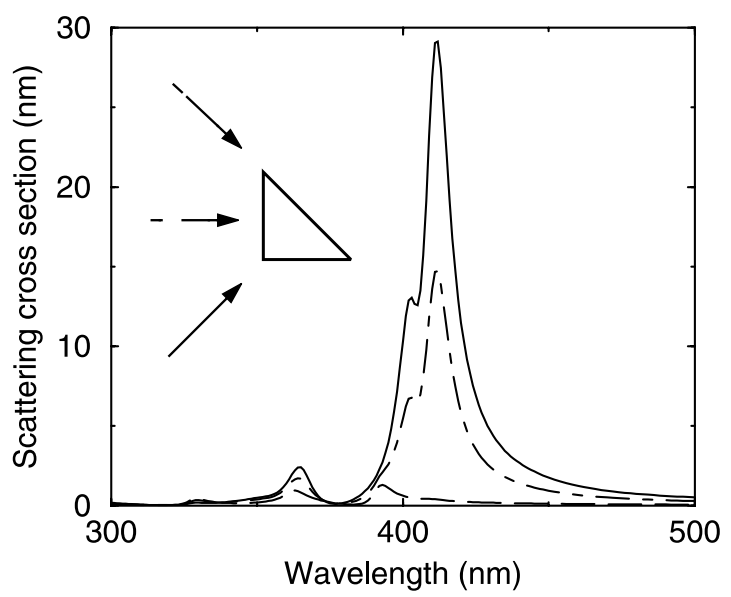

Fig. 2. Scattering cross-section for a $20 \mathrm{~nm}$ base right-angled isosceles triangular particle. Three different propagation directions for the incident field are investigated.

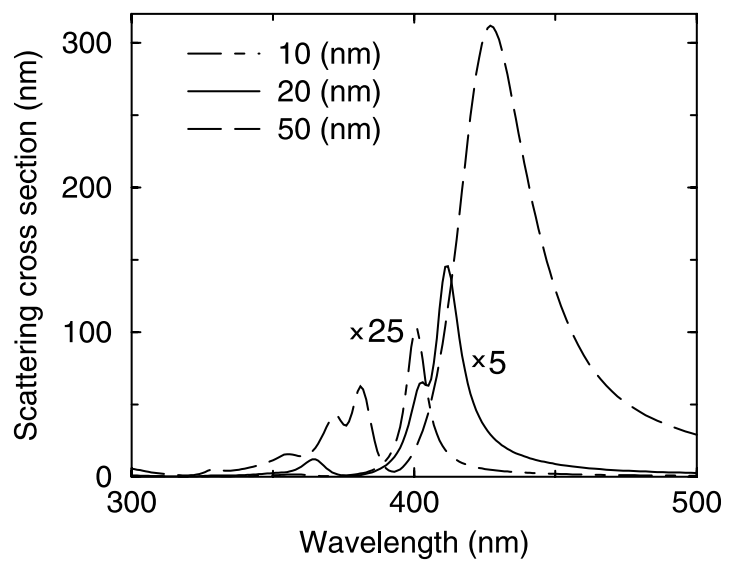

Fig. 3. Scattering cross-section for right-angled isosceles triangular particles illuminated along the (11) direction. Three different particle sizes are investigated: 10,20 and $50 \mathrm{~nm}$ base.

to $50 \mathrm{~nm}$. For cylindrical particles with similar sizes this wavelength shift amounts to only $7 \mathrm{~nm}$.

Increasing the particle size from 10 to $20 \mathrm{~nm}$ produces additional modes, including one on the short wavelength side of the main resonance (Fig. 3 ). These two modes merge into one broad resonance for the $50 \mathrm{~nm}$ particle, while several additional modes appear at shorter wavelengths, leading to a much more complex spectrum than one would obtain for a cylindrical particle of similar size.

In Fig. 4 we investigate the field distribution inside and just outside the surface of nanoparticles for selected wavelengths. A logarithmic color scale is used for the amplitude of the electric field; the amplitude of the incident electric field being one.

The data of Fig. 4a corresponds to the main resonance of a $20 \mathrm{~nm}$ particle at $\lambda=412 \mathrm{~nm}$ (see Fig. 2). Note the dramatic variations of the field in that figure: the field reaches 200 times the amplitude of the incident field at two of the corners, while vanishing at the third corner. During one half period of oscillation, large currents flow from one corner to the other as the large positive and negative polarization charges accumulate [29].

Although the incident field is oriented along the corner diagonals, the strong enhancement observed in Fig. 4a is hardly related to the lightning rod effect [28]. This is illustrated in Fig. 4b, where 

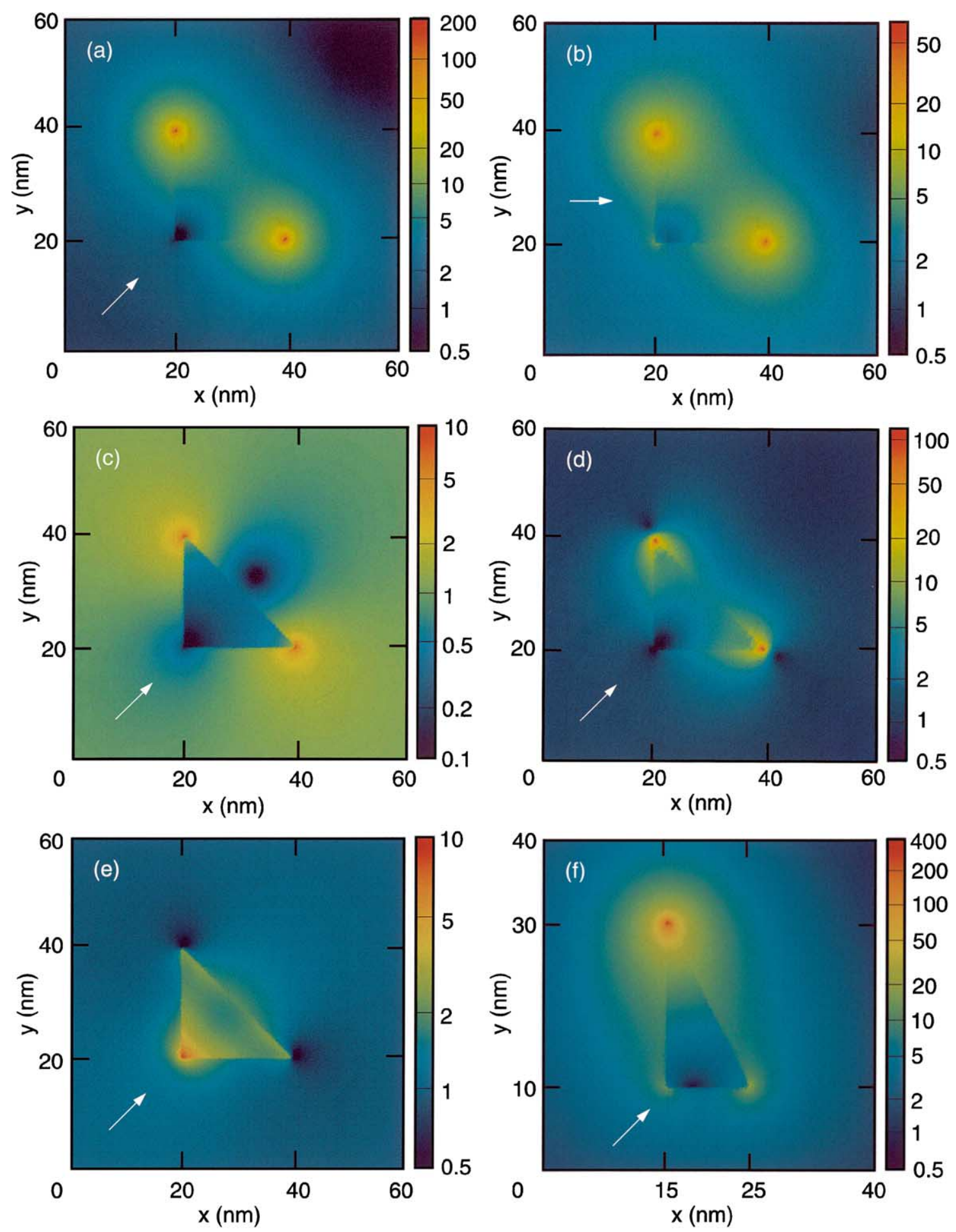
Fig. 4. Electric field amplitude distribution for triangular particles at different excitation wavelengths: (a), (b) $\lambda=412 \mathrm{~nm}$; (c) $\lambda=600$ $\mathrm{nm}$; (d) $\lambda=365 \mathrm{~nm}$; (e) $\lambda=329 \mathrm{~nm}$; (f) $\lambda=458 \mathrm{~nm}$. The propagation direction of the incident field is indicated by an arrow. (a)-(e) isosceles particles with a $20 \mathrm{~nm}$ base; (f) right-angled triangular particle with a $10 \mathrm{~nm}$ base and a 20 nm perpendicular. Logarithmic color scale.

the field distribution remains very symmetrical (i.e., dictated by the mode pattern), although the illumination direction is now different. An example which helps to set the scale of the non-resonant lightning rod contribution is illustrated in Fig. 4c, where the field distribution off-resonance at $\lambda=600 \mathrm{~nm}$ is shown. An amplitude enhancement of only 10 is obtained although the permittivity for this wavelength is strongly negative $(\varepsilon=-16.1$ $+i 0.4)$.

The field distribution in the vicinity just outside of the particle tip depends on the mode excited. While two corners radiate in all directions at $\lambda=412 \mathrm{~nm}$ (Fig. 4a), a minimum in the field is observed close to these corners at $\lambda=365 \mathrm{~nm}$ (Fig. $4 d)$. For this resonance, both plus and minus polarization charges accumulate in the corner [29], leading to a dipolar-like field distribution.

An even more peculiar behavior is obtained for $\lambda=329 \mathrm{~nm}$ (Fig. 4e). The enhancement associated with this mode is only about 10 , but quite surprisingly the field is amplified at the lower left corner, along the illumination direction. We believe this 'longitudinal' mode corresponds to the bulk plasmon since it occurs at the reflectance minimum [30]. Furthermore our results indicate that, contrary to the other modes, this resonance does not shift when the particle size increases, again indicative of a bulk rather than a surface mode.

Reducing the particle symmetry increases the field enhancement, as illustrated in Fig. $4 \mathrm{f}$ where the amplitude of the field is 400 the incident field.

To a first approximation, the enhancement of the SERS signal depends on the fourth power of the field amplitude enhancement at the molecule location [5]. We therefore computed the maximum field amplitude along the perimeter of a nanoparticle at a $1 \mathrm{~nm}$ distance from its surface. This field amplitude enhancement is presented as a function of the excitation wavelength, for three different particles of similar volume but different shape in

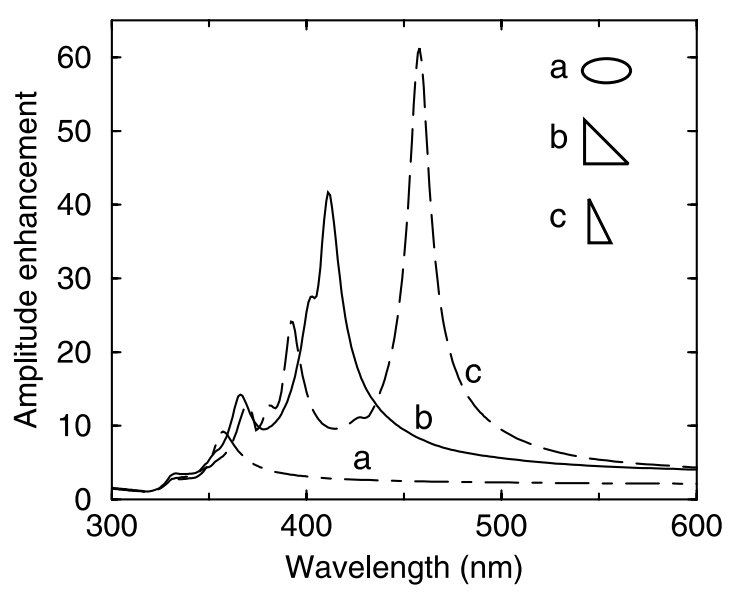

Fig. 5. Maximum field amplitude enhancement at a $1 \mathrm{~nm}$ distance from the surface of different particles: $20 \mathrm{~nm} \times 10 \mathrm{~nm}$ ellipse, $20 \mathrm{~nm}$ base isosceles triangle and $10 \mathrm{~nm}$ base, $20 \mathrm{~nm}$ perpendicular right-angled triangle. The particles are illuminated along the (1 1) direction.

Fig. 5. These data illustrate the dramatic influence of the particle shape on the enhancement. It is quite remarkable that for the $10 \mathrm{~nm}$ base rightangled triangle, the field reaches 60 times the amplitude of the incident field, corresponding to a Raman enhancement of $10^{7}$. We note also that the different resonances visible in Fig. 5 span a very large wavelength range.

It should be emphasized that the local field enhancement decreases for particles larger than 50 $\mathrm{nm}$. In the $10-50 \mathrm{~nm}$ particle range the enhancement factor for the main resonance remains within $20 \%$ of its maximum value, the latter being obtained for the $20 \mathrm{~nm}$ particle.

\section{Conclusions}

In summary, we have investigated the plasmon resonances of isolated nanowires with non-regular shapes. We have obtained a much more complex 
spectral response than for regularly shaped particles, with resonances covering a broader wavelength range. Triangular particles with dimensions smaller than $50 \mathrm{~nm}$ produce strong field enhancement near their vertices, reaching several hundred times the amplitude of the illumination wave. These strong fields are extremely localized, which can explain the hot spots and blinking phenomenon observed experimentally in Raman scattering.

\section{Acknowledgements}

This work was supported by the Swiss National Science Foundation and by the US NSF (NSFDMR-96-23949 and NSF-DMR-97-24535).

\section{References}

[1] M. Kerker, Appl. Opt. 30 (1991) 4699.

[2] M. Fleischmann, P.J. Hendra, A.J. McQuillan, Phys. Lett. 26 (1974) 163.

[3] D.L. Jeanmaire, R.P. van Duyne, J. Electroanal. Chem. 84 (1977) 1.

[4] H. Metiu, Prog. Surf. Sci. 17 (1984) 153.

[5] M. Moskovits, Rev. Mod. Phys. 57 (1985) 783.

[6] K. Kneipp, et al., Phys. Rev. Lett. 78 (1997) 1667.

[7] S. Nie, S.R. Emory, Science 275 (1997) 1102.

[8] H. Xu, E.J. Bjerneld, M. Käll, L. Börjesson, Phys. Rev. Lett. 83 (1999) 4357.

[9] A. Otto, I. Mrozek, H. Grabhorn, W. Akemann, J. Phys. C 4 (1992) 1143
[10] P. Kambhampati, C.M. Child, M.C. Foster, A. Campion, J. Chem. Phys. 108 (1998) 5013.

[11] M.I. Stockmann, et al., Phys. Rev. B 46 (1992) 2821.

[12] F.J. García-Vidal, J.B. Pendry, Phys. Rev. Lett. 77 (1996) 1163.

[13] F.J. García-Vidal, J.M. Pitarke, J.B. Pendry, Phys. Rev. B 58 (1998) 6783.

[14] N. Félidj, J. Aubard, G. Lévi, J. Chem. Phys. 111 (1999) 1195.

[15] C.F. Bohren, D.R. Huffman, Absorption and Scattering of Light by Small Particles, Wiley, New York, 1983.

[16] R. Fuchs, Phys. Rev. B 11 (1975) 1732.

[17] T.R. Jensen, G.C. Schatz, R.P. van Duyne, J. Phys. Chem. B 103 (1999) 2394.

[18] J.-C. Weeber, et al., Phys. Rev. B 60 (1999) 9061.

[19] B. Lamprecht, et al., Phys. Rev. Lett. 84 (2000) 4721.

[20] M. Kahl, et al., Sens. \& Actuators 51 (1998) 285.

[21] M. Kahl, E. Voges, Phys. Rev. B 61 (2000) 14078.

[22] M.B. Mohamed, V. Volkov, S. Link, M.A. El-Sayed, Chem. Phys. Lett. 317 (2000) 517.

[23] J. Takahara, et al., Opt. Lett. 22 (1997) 475.

[24] M. Quinten, A. Leitner, J.R. Krenn, F.R. Aussenegg, Opt. Lett. 23 (1998) 1331.

[25] P.B. Johnson, R.W. Christy, Phys. Rev. B 6 (1972) 4370.

[26] U. Kreibig, C.v. Fragstein, Z. Phys. 224 (1969) 307.

[27] J.P. Kottmann, O.J.F. Martin, IEEE Trans. Antennas Propag. 48 (2000).

[28] J. vanBladel, Singular Electromagnetic Fields and Sources, Clarendon, Oxford, 1991.

[29] J.P. Kottmann, O.J.F. Martin, D.R. Smith, S. Schultz, New J. Phys. 2 (2000) 1.

[30] R. Ruppin, in: A.D. Boardman (Ed.), Electromagnetic Surface Modes, Wiley, Chichester, 1982 (Chapter: Spherical and cylindrical surface polaritons in solids). 